\title{
Intravitreal Injection Is Associated with Increased Posterior Capsule Rupture Risk during Cataract Surgery: A Meta-Analysis
}

\author{
Zhe Zhong $^{\mathrm{a}}$ Zhenghua He $^{\mathrm{b}} \quad \mathrm{Xi} \mathrm{Yu}^{\mathrm{c}}$ Ying Zhang ${ }^{\mathrm{a}}$ \\ aDepartment of Ophthalmology, The Third Affiliated Hospital of Guangdong Medical University (Longjiang Hospital \\ of Shunde District), Foshan, China; bepartment of Otolaryngology, The Third Affiliated Hospital of Guangdong \\ Medical University (Longjiang Hospital of Shunde District), Foshan, China; ${ }^{\circ}$ Cataract Special Department, Aier Eye \\ Hospital of Yichun, Yichun, China
}

\author{
Keywords \\ Posterior capsule rupture $\cdot$ Cataract surgery $\cdot$ Intravitreal \\ therapy · Meta-analysis
}

\begin{abstract}
Background: Although observational studies have suggested that prior intravitreal therapy may predict posterior capsule rupture (PCR) during cataract surgery, this finding is still controversial. Objective: This study aimed to summarize current evidence on the association between prior intravitreal injection (IVI) and PCR during cataract surgery. Methods: A systematic literature search was performed up to October 27, 2021. Pooled odds ratios (ORs) and 95\% confidence intervals (Cls) were calculated using random-effects models. The potential association between IVI and PCR in future cataract surgeries was assessed using the following two models: "pooling the ORs of PCR in eyes with and without previous $\mathrm{IVI}(\mathrm{s})$ " and "pooling the ORs for PCR relative to each increase in the number of prior injections." The quality of included studies was appraised using the Newcastle-Ottawa Scale. Results: Six cohort studies were included in this meta-analysis, with a total of 1,051,097 eyes that underwent cataract surgery. Of these, 7,034 eyes were associated with previous
\end{abstract}

karger@karger.com www.karger.com/ore

Karger $\stackrel{\text { ' }}{5}$

BOPEN ACCESS
(C) 2021 The Author(s)

Published by S. Karger AG, Basel

This is an Open Access article licensed under the Creative Commons Attribution-NonCommercial-4.0 International License (CC BY-NC) (http://www.karger.com/Services/OpenAccessLicense), applicable to the online version of the article only. Usage and distribution for commercial purposes requires written permission.
IVI. The pooled odds of PCR in eyes with prior IVI was 2.01 (95\% Cl: 1.35-3.00) times higher than that of eyes without an IVI history. An increase in the number of previous IVI conferred increased odds of PCR of 1.03 (95\% Cl: 1.01-1.06). After excluding studies that failed to account for confounders, the significantly increased risk was not altered, and the significant heterogeneity was minimized in both models. Conclusion: This meta-analysis provides evidence that previous IVI significantly increases the risk of PCR during future cataract surgery. The risk of PCR should be discussed preoperatively with patients. Further studies are required to validate our findings and explore the underlying mechanisms.

\footnotetext{
c) 2021 The Author(s)

Published by S. Karger AG, Basel
}

\section{Introduction}

Posterior capsule rupture (PCR) is defined as unintentional communication with the posterior segment due to breach of the posterior capsule or zonular rupture, which significantly increases the risk of endophthalmitis, retinal detachment, and cystoid macular edema following cataract surgery [1]. Despite the relatively low incidence of 
complications during cataract surgery, damage to the posterior capsule is one of the most common complications and an important modifiable risk factor for visual loss after surgery [2-4].

Intravitreal injection (IVI) with antivascular endothelial growth factor (VEGF) agents has revolutionized the clinical management of multiple retinal diseases, including neovascular age-related macular degeneration (nAMD) $[5,6]$, diabetic macular edema (DME) $[7,8]$, and retinal vein occlusions (RVO) $[9,10]$. In addition, corticosteroids have anti-VEGF, anti-edematous, and anti-inflammation properties and remained a mainstay of treatment of posterior segment disorders in the era of antiVEGF [11-13]. The number of IVI has increased exponentially to become the most performed intraocular treatment worldwide. Cataract surgery on eyes with posterior segment pathology has long been debated, and inconsistent reports exist in the literature regarding the association between prior IVI and PCR during cataract surgery $[14,15]$. While Shalchi et al. [14] have reported a significantly increased risk of PCR after previous IVI, with no association with the number of injections, Lee et al. [15] reported an increased risk of PCR in eyes with at least 10 previous IVIs in a dose-dependent manner.

With the aging population and increased life expectancy, cataract surgery on patients with a history of IVI is expected to increase worldwide. Information on the potential association between IVI and cataract surgery-related complications is essential to patients and healthcare providers. Therefore, we performed a meta-analysis of observational studies to summarize current evidence to assess whether eyes with prior IVI are at a higher risk of PCR during cataract surgery.

\section{Methods}

The Preferred Reporting Items for Systematic Reviews and Meta-Analyses (PRISMA) guidelines were used while performing this meta-analysis [16]. The PRISMA guidelines are presented in online supplementary material 1 (for all online suppl. material, see www.karger.com/doi/10.1159/000521576).

\section{Literature Search}

PubMed (http://www.ncbi.nlm.nih.gov/pubmed), Embase (https://www.embase.com), and ClinicalTrials.gov (www.clinicaltrials.gov) were searched for studies with English abstracts published before October 2021, which assessed the potential relationship of prior IVI with PCR. Keywords for IVI, PCR, and cataracts surgery were combined for relevant studies (full search strategies are presented in online suppl. material 2). Manual retrieval of the reference lists was also applied to refine the search.

Risk Factor of Posterior Capsule Rupture

\section{Study Eligibility Criteria}

The following inclusion criteria were used: (1) study design: case-control or cohort studies; (2) history of IVI: there was no restriction for IVI agents, both anti-VEGFs and corticosteroids were eligible; (3) cataract procedure: extracapsular cataract extraction or phacoemulsification; (4) outcomes: studies reporting an effect estimate with $95 \%$ confidence intervals (CIs) for the relationship between prior IVI and PCR, or raw data presented in the article allowing for calculation. Exclusion criteria were (1) studies that included patients with a history of eye trauma, age under 18 years, and previous intraocular procedures other than IVI unless they provided information on subgroup analyses that met our inclusion criteria; (2) IVI of gas or air for vitreomacular interface disease; (3) abstracts from conferences, letters, and review articles. When multiple publications based on the same study were identified, the report with the most significant number of patients was used.

\section{Study Selection and Data Extraction}

Two investigators (Z.H. and Y.Z.) independently reviewed titles and abstracts of the retrieved studies to exclude irrelevant records. Later, the full-text content of the remaining articles was evaluated for inclusion. Points of disagreement in the abstract review or following selection of articles for inclusion were resolved by a discussion with the rest of the authors. The same two authors independently extracted relevant data, and any discrepancies were resolved by discussing or through arbitration by another author (X.Y.). The following data were extracted: the first author, publication year, study design, geographic regions of original studies, sample size, age, gender, indication for IVI, agents used for IVI, methods used for diagnosing PCR, the number of previous IVIs, outcomes, and confounders accounted.

\section{Quality Assessment}

We used the Newcastle-Ottawa Scale (NOS) to assess the methodological quality of studies included in the present study [17]. The NOS was developed for observational studies, consisting of three parameters of quality: selection, comparability, and outcome. The NOS assigns a maximum of 4 points for selection, 2 points for comparability, and 3 points for exposure. Studies with an NOS score of $\geq 6$ were considered eligible for inclusion. Disagreements were settled by discussion.

Data Synthesis and Analysis

The strength of association was expressed as pooled odds ratios (ORs) and corresponding 95\% CIs. The following two models were used to assess the potential relationship of IVI with the risk of PCR in future cataract surgery: "pooling the ORs of PCR in eyes with and without previous IVI" and "pooling the ORs for PCR relative to each increase in the number of prior injections." Dersimonian and Laird random-effects models, considering within-study and between-study variation, were employed to calculate pooled ORs with $95 \%$ CIs.

Methods proposed by Hamling et al. [18] were employed for studies that reported ordinal levels of the number of IVI (e.g., 1-9 previous injections, 10 or more previous injections) compared with the same reference group (i.e., eyes without previous injections) to obtain a single adjusted estimate for the risk of PCR. Software used for calculation is available at www.pnlee.co.uk/software.htm. Cochran $Q$ and $I^{2}$ statistics were used for assessing heterogeneity between studies quantitatively [19]. Publication bias was assessed by 


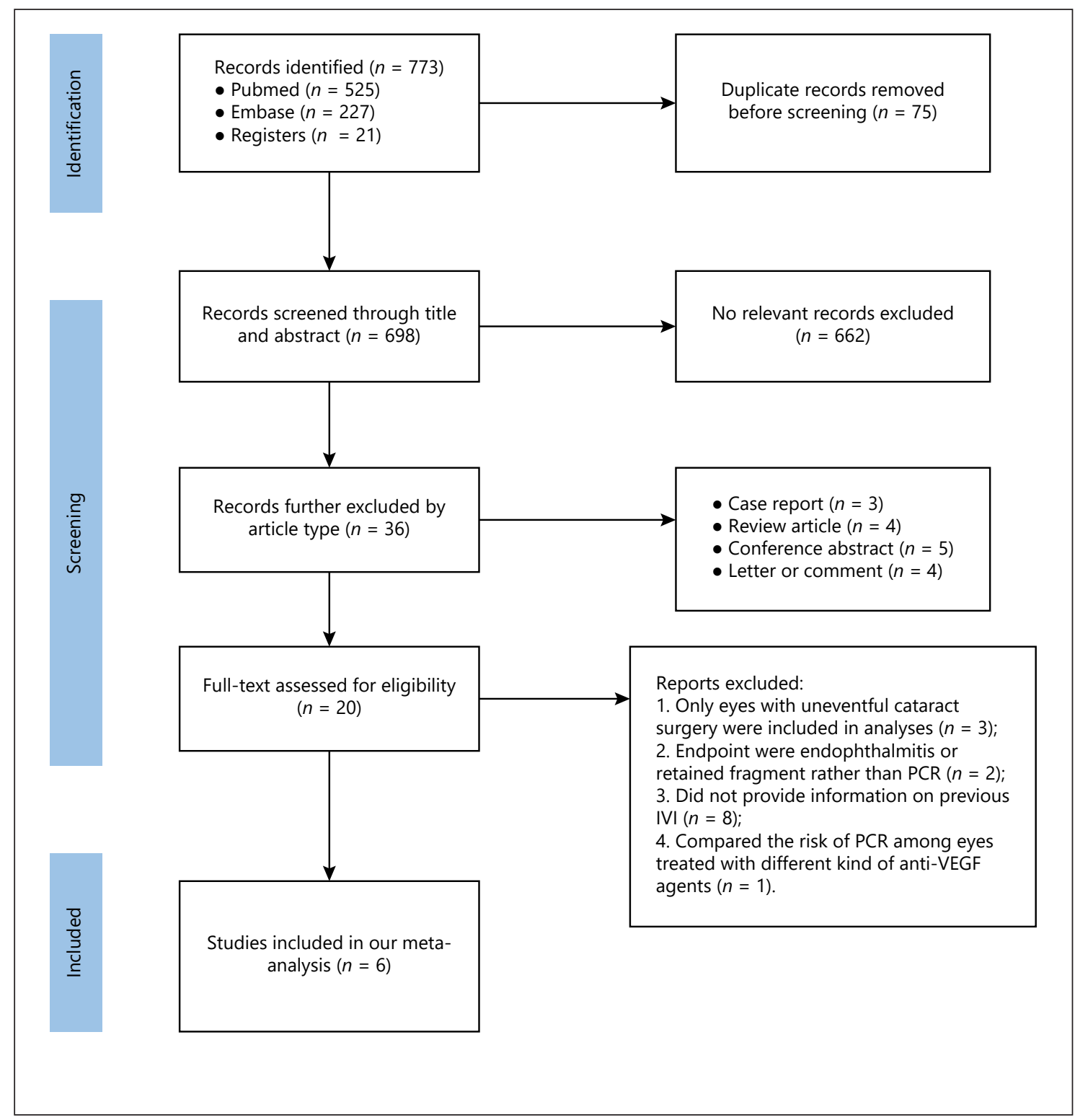

Fig. 1. Flowchart for the literature search and study selection. Anti-VEGF, antivascular endothelial growth factor; IVI, intravitreal injection; PCR, posterior capsule rupture.

inspection of funnel plots and Egger's and Begg's tests [20, 21]. A sensitivity analysis to investigate the influence of individual studies was performed by removing one study at each time. We performed post hoc subgroup analyses based on the adjusted status of studies. The risk factors for PCR included age, gender, advanced cataract, surgical grade of a surgeon, surgical difficulties, and diabetic retinopathy $[3,22,23]$. Studies that provided estimates adjusted for these confounders were included in the adjusted group. All $p$ values reported were two-sided, and those $<0.05$ were considered statistically significant. All meta-analyses were performed using Stata 13.1 (StataCorp LP, College Station, TX, USA).

\section{Results}

\section{Study Selection}

A comprehensive literature search yielded 773 relevant records, and 698 remained after deduplicating. Following the screening of titles and abstracts, 662 obviously irrelevant studies were excluded. We further excluded 16 studies because they were case reports $(n=3)$, review articles $(n=4)$, conference abstracts $(n=5)$, and letters to 

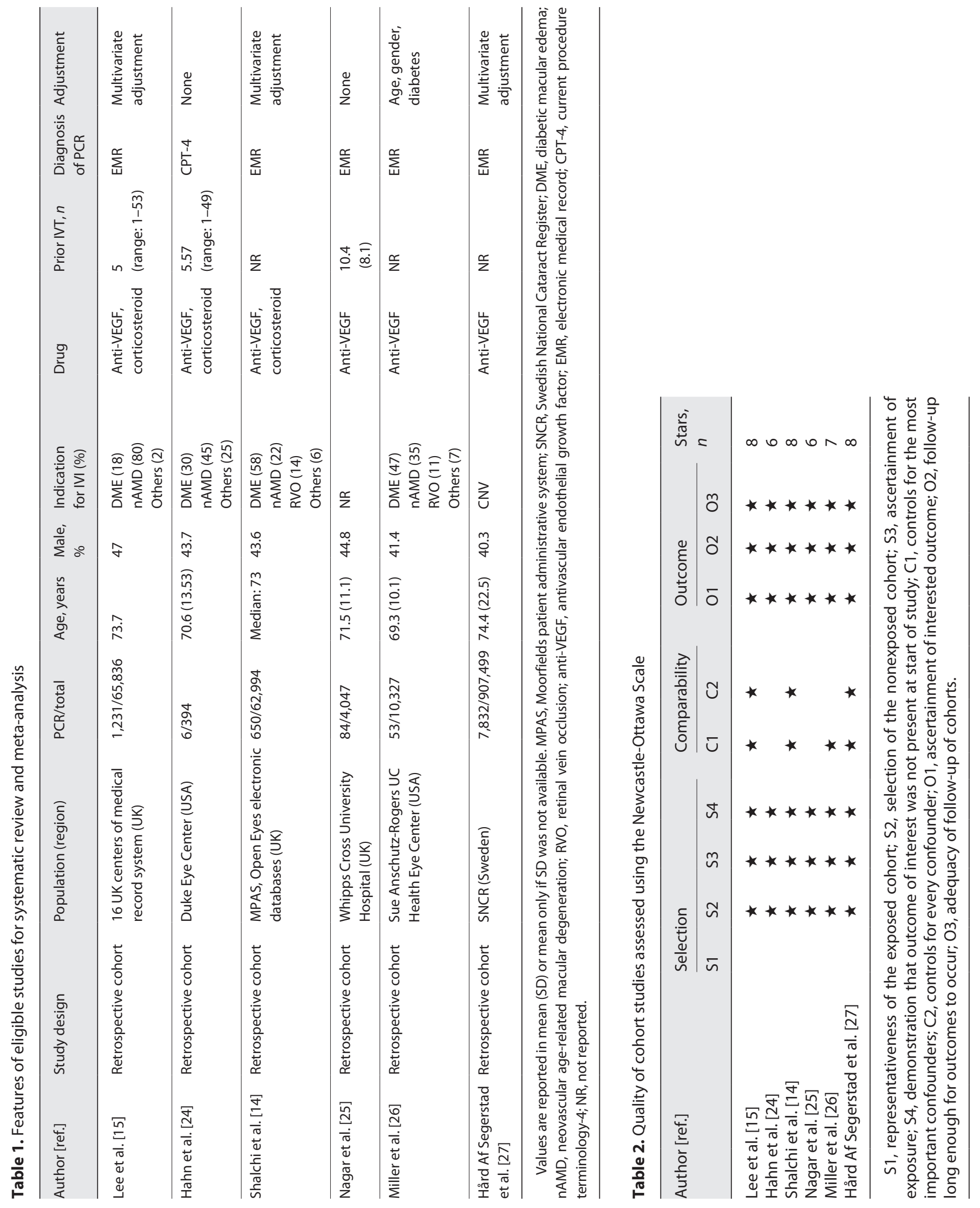
Fig. 2. Forest plot of the association between prior intravitreal injection and posterior capsule rupture. Group A: studies with multivariate-adjusted estimates. Group B: studies with crude estimates.

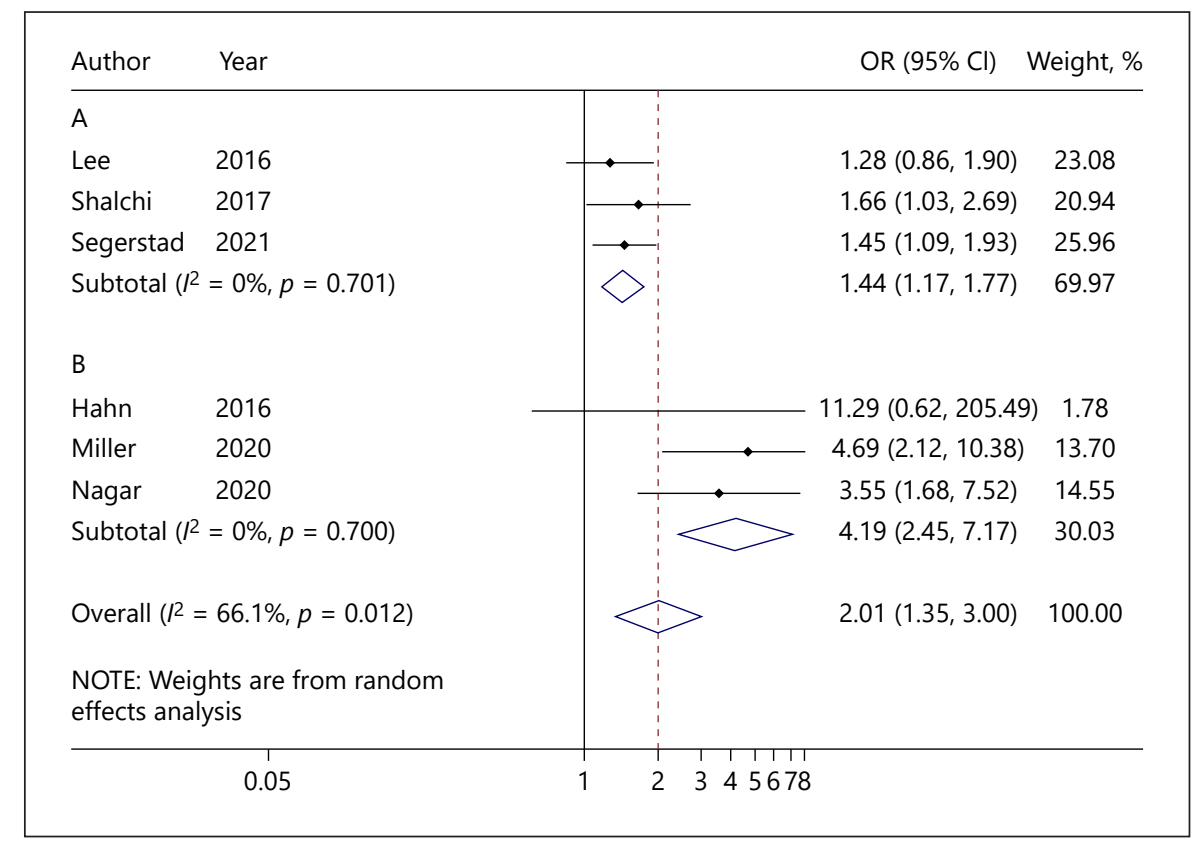

the editor or comments $(n=4)$. Accordingly, a total of 20 full-text articles were further assessed for eligibility, and 14 of them were excluded for different reasons. Ultimately, 6 articles that met the inclusion criteria were included in our meta-analysis [14, 15, 24-27]. No additional studies were identified through a manual search of the reference lists of the included studies. The process of the study selection and reasons for exclusion are detailed in Figure 1.

\section{Study Characteristics}

The characteristics of the included studies are presented in Table 1. A total of 1,051,097 eyes (range: 394907,499) underwent cataract surgery, of which 7,034 had a history of IVI. The rate of PCR was $1.41 \%(n=99)$ for eyes with previous IVI and $0.93 \%$ for those without a history of IVI $(n=9,757)$. These studies were all conducted within 5 years (2016-2021). Among the studies selected, two originated from the USA, three from the United Kingdom, and one from Sweden. All six studies were retrospective cohort studies using electronic medical records or diagnostic codes. The definition of PCR was similar in all included studies. Anti-VEGF agents were exclusively used for IVI in three studies; however, agents used for IVI included anti-VEGFs and corticosteroids in the other three studies. Indications for IVI included DME, nAMD, and RVO, among others in four studies. For the remaining two studies, choroidal neovascularization was the only indication for IVI in one study, and the indications for IVI were not mentioned in the other study. The interval between the last IVI and surgery was not provided in all studies. The methodological quality of the studies included in this meta-analysis is presented in Table 2 . The mean quality score of included studies was 7.12 (range: 6-8), indicating that the methodological quality was generally good.

\section{Previous IVIs and the Risk of PCR}

All six studies with a total of 1,051,097 surgical eyes have reported the relationship between previous IVIs and PCR. The forest plot in Figure 2 shows the association between prior IVI and PCR. The presence of prior IVI significantly increased the odds of PCR during cataract extraction (pooled OR: 2.01; 95\% CI: 1.35-3.00) with substantial heterogeneity $\left(I^{2}=66.1 \%\right.$; $\left.P_{\text {heterogeneity }}=0.012\right)$. Subgroup analysis that excluded studies with crude estimate demonstrated that the association between previous IVI and PCR remained statistically significant $(\mathrm{OR}=1.44$; $95 \% \mathrm{CI}=1.17-1.77)$ with low heterogeneity $\left(I^{2}=0 \%\right.$; $P_{\text {heterogeneity }}=0.70$ ). As shown in Figure 3, the sensitivity analyses were performed by omitting one study at a time, and the combined ORs were recalculated. Combined ORs at each time were all greater than 1 and statistically significant. Funnel plot asymmetry was observed (Fig. 4). Egger's $(p=0.046)$ but not Begg's $(p=0.133)$ test suggested the presence of publication bias. However, the re- 
Fig. 3. Sensitivity test of the association between prior intravitreal injection and posterior capsule rupture. The studies listed on the $\mathrm{Y}$ bar were omitted to see the influence of these studies on the meta-analysis results.
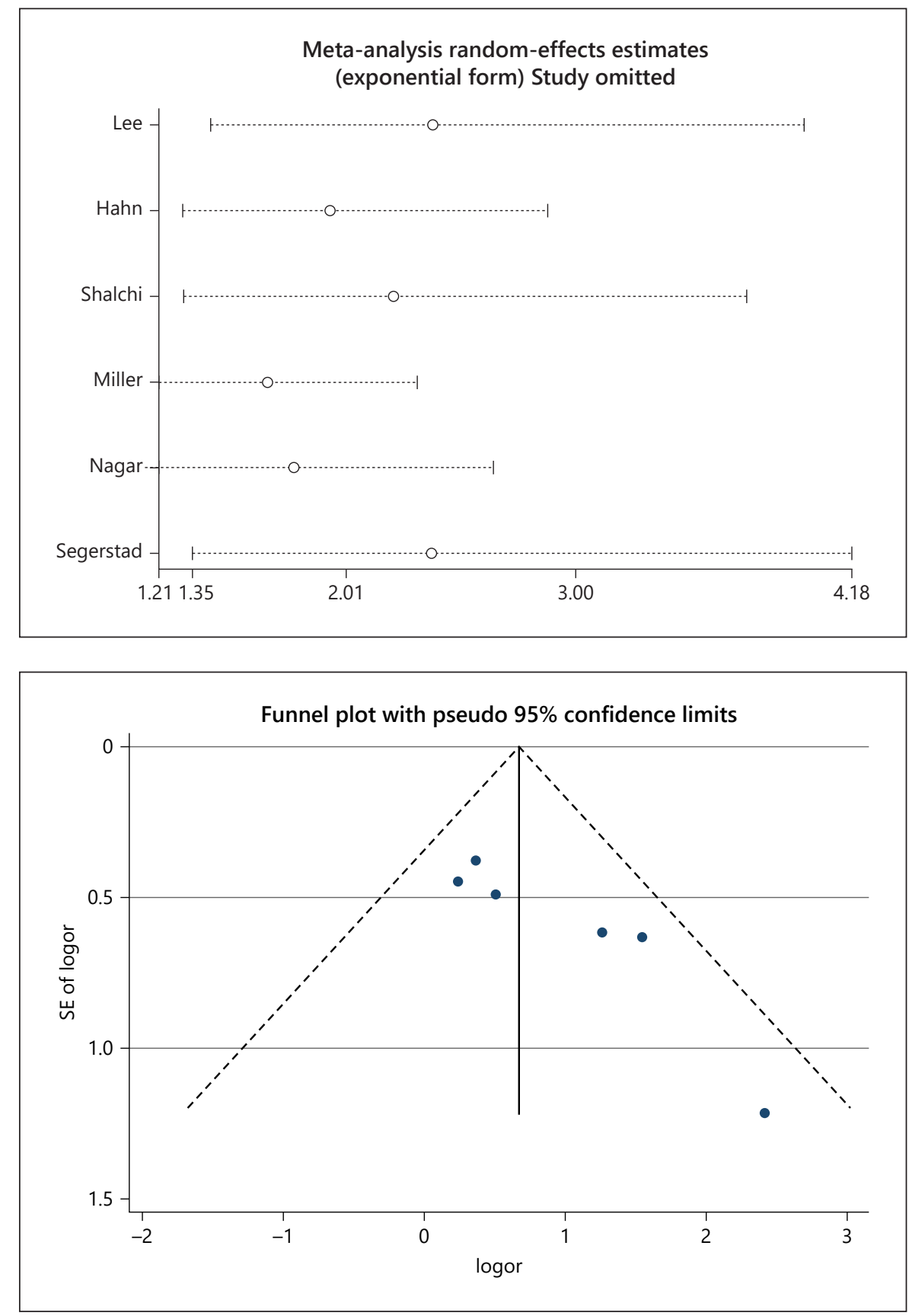

Fig. 4. Funnel plot for meta-analysis of prior intravitreal injection and the presence of posterior capsule rupture. The solid vertical line indicates pooled estimate. Dashed lines indicate a pseudo-95\% confidence interval. sults still demonstrated a significantly increased odds of PCR (OR; 1.69; 95\% CI: 1.10-2.58) after applying the trim and fill method for publication bias (Fig. 5) [28].

\section{Increase in the Number of Previous IVIs and Risk of \\ PCR}

Results from four studies that reported the relationship in a dose-response manner are shown in Figure 6. Every increase in the number of previous IVIs increased by 1.03 -times ( $95 \% \mathrm{CI}: 1.01-1.06, I^{2}=61.6 \%, P_{\text {heterogeneity }}$ $=0.050)$ the odds of PCR occurrence. The result from subgroup analysis based on three studies provided a multivariate-adjusted OR in line with the findings above (OR $=1.02 ; 95 \%$ CI: 1.01-1.04). Notably, the heterogeneity was reduced to low and had no statistical significance $\left(I^{2}\right.$ $\left.=0 \%, P_{\text {heterogeneity }}=0.556\right)$. Sensitivity analyses revealed that the effect of individual studies would not alter the results (Fig. 7). Although the funnel plot was not drawn 
Fig. 5. Funnel plot for meta-analysis of prior intravitreal injection and the presence of posterior capsule rupture after applying the trim and fill method.
Fig. 6. Forest plot of the association between the number of prior injections (per increase) and posterior capsule rupture. Group A: studies with multivariate-adjusted estimates. Group B: studies with crude estimates.

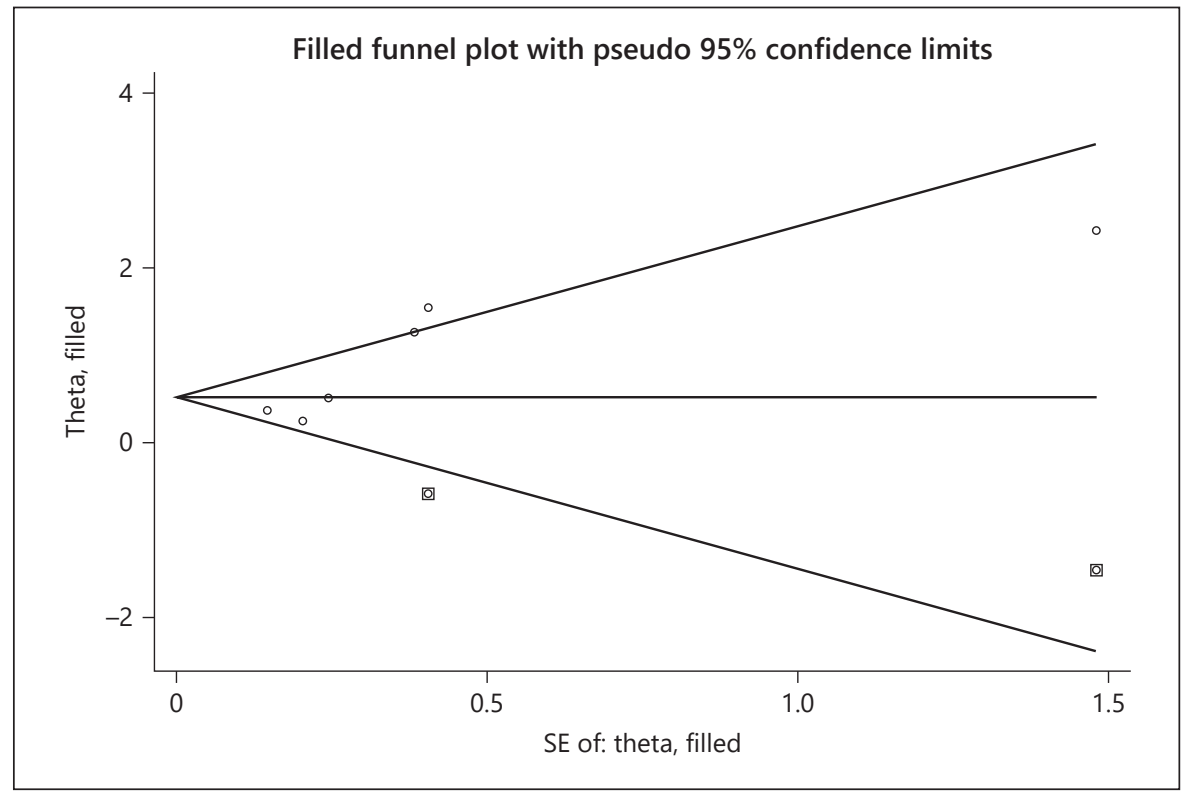

\begin{tabular}{|c|c|c|c|c|}
\hline Author & \multicolumn{2}{|l|}{ Year } & OR $(95 \% \mathrm{Cl})$ & Weight, \% \\
\hline \multicolumn{5}{|l|}{ A } \\
\hline Lee & 2016 & 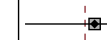 & $1.04(1.00,1.07)$ & 22.65 \\
\hline Shalchi & 2017 & 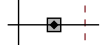 & $1.02(1.00,1.04)$ & 29.45 \\
\hline Segerstad & 2021 & 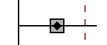 & $1.02(1.00,1.04)$ & 31.84 \\
\hline \multicolumn{2}{|c|}{ Subtotal $\left(R^{2}=0 \%, p=0.556\right)$} & $<$ & $1.02(1.01,1.04)$ & ) 83.93 \\
\hline \multicolumn{5}{|l|}{ B } \\
\hline Nagar & 2020 & & - $1.09(1.04,1.13)$ & ) 16.07 \\
\hline \multicolumn{2}{|c|}{ Subtotal $\left(R^{2}=. \%, p=.\right)$} & & $>1.09(1.04,1.13)$ & 16.07 \\
\hline \multicolumn{2}{|c|}{ Overall $\left(R^{2}=61.6 \%, p=0.050\right)$} & & $1.03(1.01,1.06)$ & 100.00 \\
\hline \multicolumn{5}{|c|}{$\begin{array}{l}\text { NOTE: Weights are from random } \\
\text { effects analysis }\end{array}$} \\
\hline & 0.881 & 1 & 1.13 & \\
\hline
\end{tabular}

due to the small number of studies, no publication bias was detected on Egger's $(p=0.086)$ or Begg's $(p=0.308)$ test.

\section{Discussion}

Although numerous eyes that undergo IVI are phakic, cataract surgery on eyes with prior IVI has not been thoroughly investigated yet. Most studies mainly focused on the effects of cataract surgery on the progression of pos- terior segment diseases and excluded eyes with intraoperative complications from their analysis [29-31]. To the best of our knowledge, this is the first meta-analysis based on observational studies that investigated the relationship between previous IVIs and PCR during cataract surgery. The results of our research suggested that the odds of PCR was 2.01 times higher in eyes with prior IVIs, and each increase in the number of previous IVIs increased the odds of PCR by 1.03-times. After excluding studies with crude estimates, this association remained statistically significant, and the marked heterogeneity was re- 
Fig. 7. Sensitivity test of the association between the number of prior injections (per increase) and posterior capsule rupture. The studies listed on the Y bar were omitted to see the influence of these studies on the meta-analysis results.

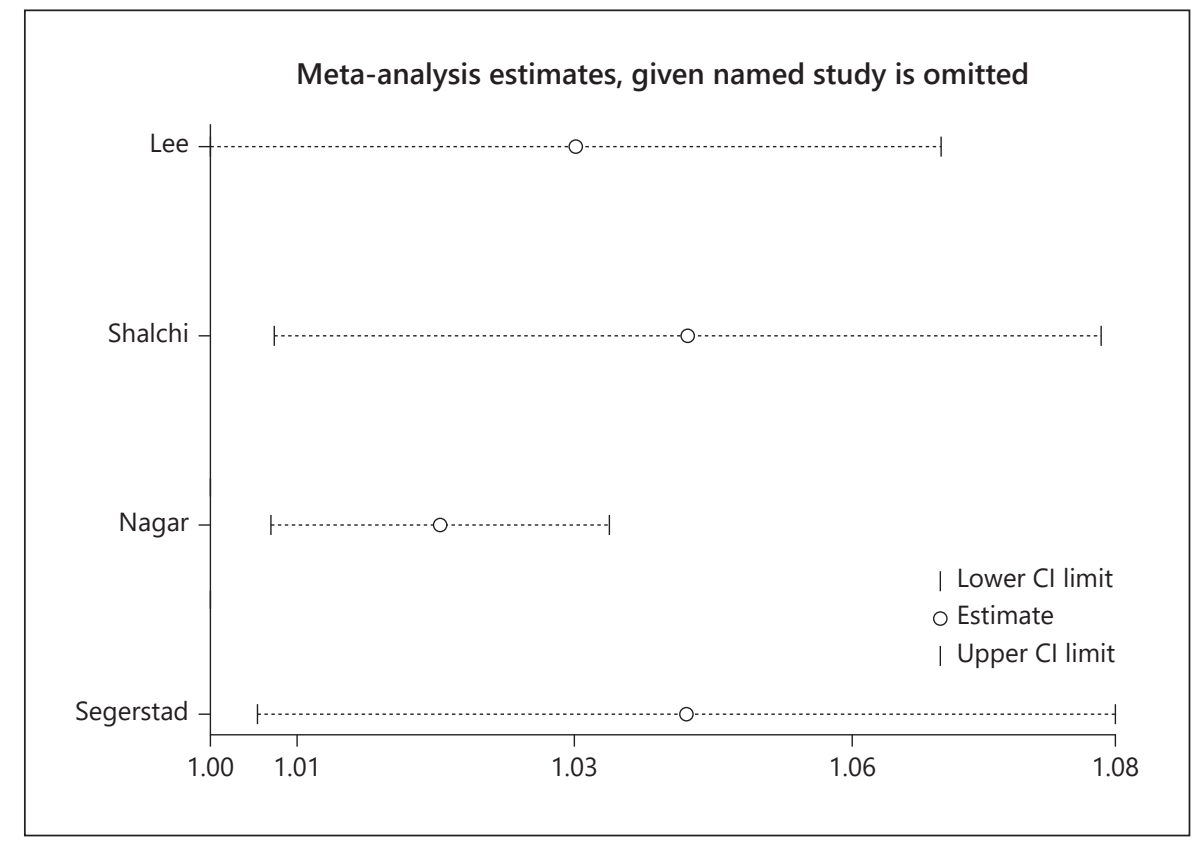

duced to low in both models. Furthermore, the robustness of our findings was confirmed by sensitivity analyses. Although the pooled OR for PCR relative to each increase in the number of previous IVIs was low (OR = 1.03), it was statistically significant (95\%: 1.01-1.06), and the majority of patients required multiple IVIs for controlling the underlying disease [32]. Therefore, we recommend that the number of prior IVIs should also be taken into account when considering the risk of PCR in eyes with prior IVIs.

The European Registry of Quality Outcomes for Cataract and Refractive Surgery (EUREQUO) is a multinational web-based registry providing data on almost 3 million cataract surgeries [33]. Using data from the EUREQUO platform, Segers et al. [34] have identified many independent risk factors for PCR. Although no information on prior IVIs was available from the EUREQUO platform, the definition of PCR, which is an intraoperative tear in the posterior capsule with or without zonular dialysis and vitreous loss, was in line with the definition used in all studies included in our meta-analysis. Compared with PCR risk estimates reported by Segers et al. [34], in the present study, the pooled likelihood of PCR for eyes with previous IVIs was greater than many other PCR predictors, including hypermetropia $(\mathrm{OR}=1.37)$, small pupil size $(\mathrm{OR}=1.23)$, white cataract $(\mathrm{OR}=1.87)$, pseudoexfoliation $(\mathrm{OR}=1.37)$, poor preoperative visual acuity $(\mathrm{OR}=1.98)$, difficult surgery including the use of hooks in the rhexis border and the use of capsule tension ring and similar devices $(\mathrm{OR}=1.37)$, age (for each additional year, $\mathrm{OR}=1.007)$, or glaucoma $(\mathrm{OR}=1.16)$. Given that previous IVIs seem to be an important risk predictor for PCR, our findings have important implications for clinical practice. It is reasonable to inform patients with prior IVIs about the increased risk of PCR. In such cases, the power of the sulcus 3-piece intraocular lens must be calculated preoperatively, and equipment for PCR management and a senior surgeon who can perform vitrectomy should be available.

The exact mechanism underlying the increased risk of PCR in eyes with prior IVIs is unclear and subject to conjecture. Several mechanisms that may be responsible for the higher PCR risk have been proposed. First of all, unidentified iatrogenic trauma to the lens during IVI may lead to PCR; subclinical lens trauma with posterior capsule tear or weakness may be quiescent until cataract surgery, which causes fluctuation of anterior chamber pressure, thus resulting in PCR [35]. Moreover, intravitreal triamcinolone has been associated with posterior subcapsular cataract formation after uncomplicated injection [36], zonular dehiscence has been observed in cases with previous ocriplasmin injection [37], and changes in lens epithelial viability and morphology after exposure to bevacizumab have been detected [38]. These classes of intravitreal agents may render lens capsules more susceptible to PCR during cataract surgery. 
The strengths of our study include the large sample size, application of quality grading, and bias assessment according to internationally accepted guidelines. Several limitations also must be acknowledged. First, the impact of the severity of the underlying retinal disorders, the interval between the last IVI and cataract surgery, and various agents used for IVI on PCR risk were not analyzed due to limited data from included studies. Second, significant publication bias in studies investigating the impact of prior IVIs on PCR was detected. Publication bias is a phenomenon whereby studies with positive results are more likely to be published than negative ones. However, a publication bias analysis of a meta-analysis, which includes fewer than 10 studies, is less reliable, which suggests that our findings might be due to chance rather than true asymmetry. Additionally, the conclusion was unchanged after adding two studies using the trim and fill method. Third, all studies were retrospective, and IVI cases and PCR identification were based on medical records or diagnostic codes. Although misclassification of PCR may occur in individual studies, there is no reason to speculate that the difference in misclassification of PCR rates between eyes with and without prior IVIs would lead to an overestimated OR of PCR in eyes with prior IVI. Finally, only six retrospective cohort studies, which are more prone to bias than randomized controlled studies, were pooled to calculate the association between prior IVIs and PCR risk. Out of six studies, only four provided data regarding the association between the number of prior IVIs and PCR, yielding less compelling evidence.

\section{Conclusion}

Our findings suggested that emphasis should be placed on preventing PCR risk when undergoing cataract surgery in eyes with a history of IVIs, especially in eyes with multiple prior IVIs. Further prospective studies with ap- propriate design are required to confirm our findings. It would be interesting to explore the impact of different agents and treatment regimens used for IVI and underlying diseases on intraoperative complications. Studies investigating the underlying mechanisms of increased PCR risk due to IVI are also required.

\section{Statement of Ethics}

All data in the present study were from previously published studies, and thus neither ethical approval nor informed consent is required. In any case, this study followed the Declaration of Helsinki completely.

\section{Conflict of Interest Statement}

No conflicts of interest to declare exist.

\section{Funding Sources}

No funds were used in the conduct and completion of this study.

\section{Author Contributions}

Z.Z. conceived the study. X.Y. performed the literature search. Z.H. and Y.Z. browsed all abstracts from the literature and extracted data from eligible articles in this meta-analysis. Z.Z. performed all statistical analyses and wrote the main manuscript text. The manuscript was scrutinized by X.Y. The present manuscript was approved by all authors.

\section{Data Availability Statement}

All data in this article were based on studies that have already been published.

\section{References}

1 Day AC, Donachie PH, Sparrow JM, Johnston RL. The Royal College of Ophthalmologists' National Ophthalmology Database study of cataract surgery: report 1, visual outcomes and complications. Eye. 2015 Apr;29(4):55260.

2 Sparrow JM, Taylor H, Qureshi K, Smith R, Birnie K, Johnston RL. The Cataract National Dataset electronic multi-centre audit of 55,567 operations: risk indicators for monocular visual acuity outcomes. Eye. 2012 Jun; 26(6):821-6.
3 Salowi MA, Chew FLM, Adnan TH, King C, Ismail M, Goh PP. The Malaysian Cataract Surgery Registry: risk indicators for posterior capsular rupture. Br J Ophthalmol. 2017 Nov; 101(11):1466-70.

4 Zetterberg M, Kugelberg M, Nilsson I, Lundström M, Behndig A, Montan P. A composite risk score for capsule complications based on data from the Swedish National Cataract Register: relation to surgery volumes. Ophthalmology. 2021 Mar;128(3):364-71.
5 Brown DM, Michels M, Kaiser PK, Heier JS, Sy JP, Ianchulev T. Ranibizumab versus verteporfin photodynamic therapy for neovascular age-related macular degeneration: two-year results of the ANCHOR study. Ophthalmology. 2009 Jan;116(1):57-65.e5.

6 Rosenfeld PJ, Brown DM, Heier JS, Boyer DS, Kaiser PK, Chung CY, et al. Ranibizumab for neovascular age-related macular degeneration. N Engl J Med. 2006 Oct 5;355(14):141931. 
7 Wells JA, Glassman AR, Ayala AR, Jampol LM, Aiello LP, Antoszyk AN, et al. Aflibercept, bevacizumab, or ranibizumab for diabetic macular edema. N Engl J Med. $2015 \mathrm{Mar}$ 26;372(13):1193-203.

8 Nguyen QD, Brown DM, Marcus DM, Boyer DS, Patel S, Feiner L, et al. Ranibizumab for diabetic macular edema: results from 2 phase III randomized trials - RISE and RIDE. Ophthalmology. 2012 Apr;119(4):789-801.

9 Brown DM, Campochiaro PA, Singh RP, Li Z, Gray S, Saroj N, et al. Ranibizumab for macular edema following central retinal vein occlusion: six-month primary end point results of a phase III study. Ophthalmology. 2010 Jun; 117(6):1124-33.e1.

10 Schmidt-Erfurth U, Garcia-Arumi J, Gerendas BS, Midena E, Sivaprasad S, Tadayoni R, et al. Guidelines for the management of retinal vein occlusion by the European Society of Retina Specialists (EURETINA). Ophthalmologica. 2019;242(3):123-62.

11 Martidis A, Duker JS, Greenberg PB, Rogers AH, Puliafito CA, Reichel E, et al. Intravitreal triamcinolone for refractory diabetic macular edema. Ophthalmology. 2002 May; 109(5): 920-7.

12 Gillies MC, Lim LL, Campain A, Quin GJ, Salem W, Li J, et al. A randomized clinical trial of intravitreal bevacizumab versus intravitreal dexamethasone for diabetic macular edema: the BEVORDEX study. Ophthalmology. 2014 Dec;121(12):2473-81.

13 Nghiem-Buffet S, Baillif S, Regnier S, Skelly A, Yu N, Sodi A. Response to comment on: treatment patterns of ranibizumab intravitreal injection and dexamethasone intravitreal implant for retinal vein occlusion in the USA. Eye. 2017 Apr;31(4):1113-4.

14 Shalchi Z, Okada M, Whiting C, Hamilton R. Risk of posterior capsule rupture during cataract surgery in eyes with previous intravitreal injections. Am J Ophthalmol. 2017 May;177: $77-80$.

15 Lee AY, Day AC, Egan C, Bailey C, Johnston RL, Tsaloumas MD, et al. Previous intravitreal therapy is associated with increased risk of posterior capsule rupture during cataract surgery. Ophthalmology. 2016 Jun;123(6): $1252-6$.

16 Liberati A, Altman DG, Tetzlaff J, Mulrow C, Gøtzsche PC, Ioannidis JP, et al. The PRISMA statement for reporting systematic reviews and meta-analyses of studies that evaluate health care interventions: explanation and elaboration. PLoS Med. 2009 Jul 21;6(7): e1000100.

17 Wells G. The Newcastle-Ottawa Scale (NOS) for assessing the quality of nonrandomised studies in meta-analyses. Appl Eng Agric. 2014;18(6):727-34.

18 Hamling J, Lee P, Weitkunat R, Ambühl M. Facilitating meta-analyses by deriving relative effect and precision estimates for alternative comparisons from a set of estimates presented by exposure level or disease category. Stat Med. 2008 Mar 30;27(7):954-70.

19 Higgins JP, Thompson SG, Deeks JJ, Altman DG. Measuring inconsistency in meta-analyses. BMJ. 2003 Sep 6;327(7414):557-60.

20 Egger M, Davey Smith G, Schneider M, Minder C. Bias in meta-analysis detected by a simple, graphical test. BMJ. 1997 Sep 13; 315(7109):629-34.

21 Begg CB, Mazumdar M. Operating characteristics of a rank correlation test for publication bias. Biometrics. 1994 Dec;50(4):1088-101.

22 Ramsell TG. Lens capsule in diabetes. $\mathrm{Br}$ J Ophthalmol. 1969 Feb;53(2):98-100.

23 Ridderskär L, Montan P, Kugelberg M, Nilsson I, Lundström M, Behndig A, et al. Outcome of cataract surgery in eyes with diabetic retinopathy: a Swedish national cataract register report. Acta Ophthalmol. 2021 May 27. Epub ahead of print.

24 Hahn P, Jiramongkolchai K, Stinnett S, Daluvoy M, Kim T. Rate of intraoperative complications during cataract surgery following intravitreal injections. Eye. 2016 Aug;30(8): $1101-9$.

25 Nagar AM, Luis J, Kainth N, Panos GD, McKechnie CJ, Patra S. The risk of posterior capsule rupture during phacoemulsification cataract surgery in eyes with previous intravitreal anti vascular endothelial growth factor injections. J Cataract Refract Surg. 2020 Feb; 46(2):204-8.

26 Miller DC, Christopher KL, Patnaik JL, Lynch AM, Seibold LK, Mandava N, et al. Posterior capsule rupture during cataract surgery in eyes receiving intravitreal antiVEGF injections. Curr Eye Res. 2021 Feb; 46(2):179-84.

27 Hård Af Segerstad P. Risk model for intraoperative complication during cataract surgery based on data from 900,000 eyes: previous intravitreal injection is a risk factor. $\mathrm{Br} \mathrm{J}$ Ophthalmol. 2021 Apr 22. Epub ahead of print.

28 Duval S, Tweedie R. A nonparametric "trim and fill" method of accounting for publication bias in meta-analysis. J Am Stat Assoc. 2000; 95(449):89-98.

29 Tham YC, Liu L, Rim TH, Zhang L, Majithia S, Chee ML, et al. Association of Cataract Surgery with risk of diabetic retinopathy among Asian participants in the Singapore Epidemiology of Eye Diseases Study. JAMA Netw Open. 2020 Jun 1;3(6):e208035.

30 Wang JJ, Fong CS, Burlutsky G, Cugati S, Tan AG, Rochtchina E, et al. Risk of age-related macular degeneration 4 to 5 years after cataract surgery. Ophthalmology. 2016 Aug; 123(8):1829-30.e1.

31 Bek T, Tilma K, la Cour M. The risk for developing vision-threatening retinopathy after cataract surgery in diabetic patients depends on the postoperative follow-up time. Acta Ophthalmol. 2021 Aug 2. Epub ahead of print.

32 Fernández-Robredo P, Sancho A, Johnen S, Recalde S, Gama N, Thumann G, et al. Current treatment limitations in age-related macular degeneration and future approaches based on cell therapy and tissue engineering. J Ophthalmol. 2014;2014:510285.

33 Lundström M, Barry P, Brocato L, Fitzpatrick C, Henry Y, Rosen P, et al. European registry for quality improvement in cataract surgery. Int J Health Care Qual Assur. 2014;27(2): 140-51.

34 Segers MH, Behndig A, van den Biggelaar FJ, Brocato L, Henry YP, Nuijts RM, et al. Risk factors for posterior capsule rupture in cataract surgery as reflected in the European Registry of Quality Outcomes for Cataract and Refractive Surgery. J Cataract Refract Surg. 2022 Jan 1;48(1):51-5.

35 Khalifa YM, Pantanelli SM. Quiescent posterior capsule trauma after intravitreal injection: implications for the cataract surgeon. J Cataract Refract Surg. 2011 Jul;37(7):1364.

36 Thompson JT. Cataract formation and other complications of intravitreal triamcinolone for macular edema. Am J Ophthalmol. 2006 Apr;141(4):629-37.

37 Keller J, Haynes RJ. Zonular dehiscence at the time of combined vitrectomy and cataract surgery after intravitreal ocriplasmin injection. JAMA Ophthalmol. 2015 Sep;133(9): 1091-2.

38 Jun JH, Sohn WJ, Lee Y, Kim JY. Effects of anti-vascular endothelial growth factor monoclonal antibody (bevacizumab) on lens epithelial cells. Clin Ophthalmol. 2016;10: 1167-74. 